\title{
Phase Separation in the Two-Dimensional Hubbard Model
}

\author{
Gang Su* \\ Institut für Theoretische Physik, Universität zu Köln \\ Zülpicher Strasse 77, D-50937 Köln, Germany \\ (Received 30 May 1996, Phys. Rev. B54, 8281R(1996))
}

\begin{abstract}
By making use of known exact results and symmetry properties for the one-band Hubbard model, we show in a somewhat exact manner that there is no phase separation on a square lattice at arbitrary fillings at finite temperature for both attractive and repulsive on-site Coulomb interaction. This result is consistent with the quantum Monto Carlo calculation.
\end{abstract}

PACS numbers: 71.27.+a, 74.80.-g

The considerable experimental evidence shows that $\mathrm{La}_{2} \mathrm{CuO} \mathrm{O}_{4+\delta}$ has a regime in which phase separation occurs [1]. Such a separation appears between a phase very close to $\mathrm{La}_{2} \mathrm{CuO}_{4}$ (i.e., an oxygen-poor phase) and an oxygen-rich phase that becomes superconducting at $T_{c} \sim 38 \mathrm{~K}$. Later on, this phenomenon was actively investigated and confirmed in many other high Tc superconductors [2]. People therefore believe that the presence of phase separation is of essential importance for understanding the physics of the cuprate superconductors [3]. Apart from the experimental works, there are also some theoretical investigations devoting to this subject, see Refs. 2, . 1 for excellent reviews. As most of theoretical studies, being based on the planar t-J and Hubbard models, are numerical or approximate, the obtained results still remain controversial [4,2]. Quite recently, Au et al. [5], using the symmetry properties and known exact results obtained by Lieb [6], Kubo and Kishi [7] for the Hubbard model, got an exact result on phase separation in the Hubbard model on bipartite lattices. They asserted that there is no phase separation at low dopings at any temperature for the repulsive Hubbard model. This exact consequence clarifies, to some extent, the existing controversy in the Hubbard model and in the t-J model with small values of $\mathrm{J} / \mathrm{t}$. However, several issues concerning this problem still remain to be addressed in the sense that the exact results are sparse. For instance, if phase separation exactly occurs in the cases with moderate or even high dopings or in the attractive (negative-U) Hubbard model on symmetric bipartite lattices (e.g. on square lattices or cubic lattices), is still inconclusive. Since there have been numerical results indicating no phase separation for the one-band Hubbard model on square lattices at any fillings [8, 4, it would be quite interesting to seek more exact evidences supporting this observation.

In this note, making use of known exact results obtained by Ghosh [9] and symmetry properties for the one-band Hubbard model on a square lattice, we shall show that there is no phase separation at any fillings at finite temperature for both attractive and repulsive onsite Coulomb interaction. This statement, being exact, is consistent with the quantum Monto Carlo calculation
8, 何.

We start from the one-band Hubbard model in an external field $h$ on a square lattice with $M$ sites. The Hamiltonian reads

$$
\begin{aligned}
& H=-t \sum_{<i, j>\sigma}\left(c_{i \sigma}^{\dagger} c_{j \sigma}+h . c .\right)+U \sum_{i} n_{i \uparrow} n_{i \downarrow} \\
& -\mu \sum_{i}\left(n_{i \uparrow}+n_{i \downarrow}\right)-\frac{h}{2} \sum_{i}\left(n_{i \uparrow}-n_{i \downarrow}\right),
\end{aligned}
$$

where the notations are standard. The foregoing discussion is independent of the sign of $U$, and is only valid for finite temperatures and for the finite chemical potential. In the following, we shall first investigate the phase separation near half-filling, and then discuss the problem away from half-filling. The advantage of our method is the fact that we can treat the problem in a unifying way for both $U>0$ and $U<0$.

The method which we shall adopt for the case near half-filling, being based on the well-known particle-hole transformation, is very similar to that exploited in Ref. [5], but we do not invoke any a priori assumption. For the Hamiltonian (1) defined on a square lattice, Ghosh [9] obtained, for small $h$, an exact upper bound

$$
|m(h, T)| \leq \frac{\text { const. }}{T^{\frac{1}{2}}} \frac{1}{\left.|\ln | h\right|^{\frac{1}{2}}},
$$

where $m(h, T)=\frac{1}{M} \sum_{i}\left\langle S_{i}^{z}\right\rangle$, the magnetization per site, and $\langle\cdots\rangle$ is the thermal average over a grand canonical ensemble. As $h \rightarrow 0,|m(h, T)| \rightarrow 0$ means the absence of spontaneous magnetic long-range order, implying that the system could exhibit paramagnetic behaviors at temperature $T>0$. For small $h$, inequality (2) ensures the analyticity (only in the sense that the first derivative exists) of $m(h, T)$. Therefore, we can conclude that $m(h, T>0)$ is continuous and analytic in the neighborhood of $h=0$.

We now apply the unitary particle-hole transformation,

$$
c_{i \uparrow} \rightarrow c_{i \uparrow}, \quad c_{i \downarrow} \rightarrow \epsilon(i) c_{i \downarrow}^{\dagger},
$$

with $\epsilon(i)=-1$ for $i \in$ the one sublattice and -1 for $i \in$ the other sublattice, to the Hamiltonian (1), and obtain 


$$
\begin{aligned}
& H^{\prime}=-t \sum_{<i, j>\sigma}\left(c_{i \sigma}^{\dagger} c_{j \sigma}+h . c .\right)+U^{\prime} \sum_{i} n_{i \uparrow} n_{i \downarrow} \\
& -\mu^{\prime} \sum_{i}\left(n_{i \uparrow}+n_{i \downarrow}\right)-\frac{h^{\prime}}{2} \sum_{i}\left(n_{i \uparrow}-n_{i \downarrow}\right),
\end{aligned}
$$

with

$$
U^{\prime}=-U, \quad \mu^{\prime}=\frac{h-U}{2}, \quad h^{\prime}=2 \mu-U,
$$

where a constant term is dropped. Eq.(2) is transformed into

$$
\begin{aligned}
& 1-\frac{\text { const. }}{T^{1 / 2}} \frac{1}{|\ln | U^{\prime}-2 \mu^{\prime}||^{1 / 2}} \leq \rho\left(\mu^{\prime}, T\right) \\
& \leq 1+\frac{\text { const. }}{T^{1 / 2}} \frac{1}{|\ln | U^{\prime}-2 \mu^{\prime}||^{1 / 2}}
\end{aligned}
$$

for small $\left|U^{\prime}-2 \mu^{\prime}\right|$, where $\rho=\frac{1}{M} \sum_{i}\left\langle n_{i \uparrow}+n_{i \downarrow}\right\rangle$, the electron density per site. Inequality (6) is of basic importance. For the system described by the one-band Hubbard model (4) on the square lattice, the density $\rho\left(\mu^{\prime}, T>0\right)$ is thus the continuous function of the chemical potential $\mu^{\prime}$ near $\frac{U^{\prime}}{2}$, i.e., at small doping (note that at half-filling, $\mu^{\prime}=\frac{U^{\prime}}{2}$ ). This can be justified by noting the fact $\lim _{\delta \rightarrow 0} \rho\left(\mu^{\prime}=U^{\prime} / 2+\delta, T>0\right)=\lim _{\delta \rightarrow 0} \rho\left(\mu^{\prime}=\right.$ $\left.U^{\prime} / 2-\delta, T>0\right)$. An alternative criterion for phase separation is based on this fact [4: if a discontinuity is found in $\rho\left(\mu^{\prime}, T\right)$ as a function of $\mu^{\prime}$, then the densities inside the gap are unstable, giving rise to a phase-separated state, and if it is not found, then no phase separation occurs. Actually, phase separation falls in the class of the first-order phase transition, as the first-order phase transition into two phases with different densities is featured by the discontinuity of $\rho\left(\mu^{\prime}, T\right)$. Thus the continuity of $\rho\left(\mu^{\prime}, T>0\right)$ near $U^{\prime} / 2$ suggests that the one-band Hubbard model on the square lattice at $T>0$ does not exhibit phase separation at small doping (near half-filling), which is consistent with the quantum Monto Carlo calculation [8,4] and with the result of high-temperature expansion for the two dimensional t-J model with small values of $J / t$ [10 which can be viewed as the strongly coupling limit of the Hubbard model. As in the beginning we do not fix the sign of $U$, this exact result remains true for both negative and positive $U^{\prime}$ as well as the vanishing external field $\left(h^{\prime}=0\right)$. If the system is indeed paramagnetic, as being plausibly expected, then this result could be extended to moderate dopings. One may observe that this approach only works for the two dimensions (or the one dimension) but not for the three dimensions, as the Ghosh's results were obtained only for the low dimensional cases.

Now let us look at this problem when the system is doped away from half-filling. We introduce the $\eta$ pairing (or pseudospin) operators [6,11] as $\eta^{+}=\sum_{i} \epsilon(i) c_{i \uparrow}^{\dagger} c_{i \downarrow}^{\dagger}$, $\eta^{-}=\left(\eta^{+}\right)^{\dagger}$, and $\eta^{z}=\frac{1}{2}(N-M)$ with $N=\sum_{i}\left(n_{i \uparrow}+n_{i \downarrow}\right)$. They obey the usual $S U(2)$ Lie algebra. We from now on set $h=0$ in Eq.(1). As a matter of fact, $\left[\eta^{-}, H\right]=$ $2\left(\mu_{c}-\mu\right) \eta^{-}$, where $\mu_{c}=\frac{U}{2}$. Using this commutator and the cyclicity under the trace we obtain 12]

$$
F(\mu, T)\left\{1-\exp \left[2 \beta\left(\mu_{c}-\mu\right)\right]\right\}=\rho(\mu, T)-1,
$$

with $F(\mu, T)=\frac{1}{M}\left\langle\eta^{+} \eta^{-}\right\rangle$, and $\beta$ the inverse temperature. Since the right-hand side of (7) is an intensive quantity, $F(\mu, T)$, being obviously non-negative, should also be intensive. Furthermore, it is well-known [13] that, based on the particle-hole transformation, $\rho(\mu, T)=1$ (half-filling) as $\mu=\mu_{c}$ and vice versa. Consequently, combining this result and Eq.(7) we get the following relation

$$
\begin{aligned}
& \rho(\mu, T)>1 \text { as } \mu>\mu_{c}, \quad \rho(\mu, T)=1 \text { as } \mu=\mu_{c}, \\
& \text { and } \rho(\mu, T)<1 \text { as } \mu<\mu_{c} .
\end{aligned}
$$

This exact constraint may have some implications. First, a direct consequence is $F(\mu, T)>0$ for $\mu \neq \mu_{c}$. Second, since $\rho(\mu, T)$ is analytic in the neighborhood of $\mu=\mu_{c}$ (ensured by (6)), at small doping, we can expand $\rho(\mu, T)$ in powers of $\left(\mu-\mu_{c}\right)$, obtaining

$\rho(\mu, T)=1+\left.\frac{\partial \rho(\mu, T)}{\partial \mu}\right|_{\mu=\mu_{c}}\left(\mu-\mu_{c}\right)+O\left[\left(\mu-\mu_{c}\right)^{2}\right]$,

where we have used $\rho\left(\mu=\mu_{c}, T\right)=1$. Up to the second order in $\left(\mu-\mu_{c}\right)$, we see that $\left.\frac{\partial \rho(\mu, T)}{\partial \mu}\right|_{\mu=\mu_{c}}=$ finite $>0$ (otherwise it contradicts (6) and (8)).

Since the functions $\rho(\mu, T)$ and $F(\mu, T)$ are closely related, let us now study the properties of the latter. From Eq.(7) we know that $F(\mu, T)$ is finite, and comply

$$
0<F(\mu, T) \leq \frac{1}{\left|1-\exp \left[2 \beta\left(\mu_{c}-\mu\right)\right]\right|}
$$

for $\mu \neq \mu_{c}$. Furthermore, in accordance with the definition of $F(\mu, T)$, we have

$$
F(\mu, T) \equiv \frac{1}{M} \frac{\operatorname{Tr}\left[e^{-\beta\left(H_{0}-\mu N\right)} \eta^{+} \eta^{-}\right]}{T r e^{-\beta\left(H_{0}-\mu N\right)}},
$$

where we have denoted Eq.(1) by $H=H_{0}-\mu N$ (Recall that $h=0)$. Employing the following symmetric particlehole transformation,

$$
c_{i \uparrow}^{\dagger} \rightarrow \epsilon(i) c_{i \uparrow}, \quad c_{i \downarrow}^{\dagger} \rightarrow \epsilon(i) c_{i \downarrow},
$$

we find $H \rightarrow \tilde{H}=H_{0}-(U-\mu) N+(U-2 \mu) M$, and $\eta^{+} \eta^{-} \rightarrow \eta^{-} \eta^{+}$. By applying the unitary transformation, Eq.(12), to $F(\mu, T)$, we obtain

$$
\begin{aligned}
& F(\mu, T)=\frac{1}{M} \frac{\operatorname{Tr}\left\{e^{-\beta\left[H_{0}-(U-\mu) N\right]} \eta^{-} \eta^{+}\right\}}{\operatorname{Tr} e^{-\beta\left[H_{0}-(U-\mu) N\right]}} \\
& =F\left(2 \mu_{c}-\mu, T\right) e^{2 \beta\left(\mu-\mu_{c}\right)}
\end{aligned}
$$

where we have used $\eta^{-} \eta^{+}=\eta^{+} \eta^{-}-2 \eta^{z}$, the definition of $F(\mu, T)$, as well as Eq.(7). Eq.(13) completely determines the form of $F(\mu, T)$. As $\mu^{\prime}=\mu_{c}-\mu$, it becomes 
$F\left(\mu_{c}-\mu, T\right)=F\left(\mu_{c}+\mu, T\right) \exp (-2 \beta \mu)$, which reflects the symmetry of $F(\mu, T)$ as a function of $\mu$. Similarly, we can obtain, after operating the transformation (12) to $\rho(\mu, T)$, the following equation

$$
\rho(\mu, T)=2-\rho\left(2 \mu_{c}-\mu, T\right)
$$

One may verify that Eqs.(7), (13) and (14) are selfconsistent.

By differentiating Eq.(11) with respect to $\mu$, and using the unitary particle-hole transformation (12) to the thermal averages involved, and then noting Eqs.(7) and (13), one can prove exactly the following expression

$$
\begin{aligned}
& \frac{\partial F(\mu, T)}{\partial \mu}-\frac{\partial F\left(2 \mu_{c}-\mu, T\right)}{\partial \mu} \\
& =\frac{\beta}{M}\left(\left\langle N^{2}\right\rangle-\langle N\rangle^{2}\right)_{2 \mu_{c}-\mu, T},
\end{aligned}
$$

where $\langle\cdots\rangle_{\mu, T}$ means the thermal average with the chemical potential $\mu$ at temperature $T$. Considering $\left\langle N^{2}\right\rangle-$ $\langle N\rangle^{2} \geq 0$, we get $\frac{\partial F(\mu, T)}{\partial \mu} \geq \frac{\partial F\left(2 \mu_{c}-\mu, T\right)}{\partial \mu}$. By (14) and (10), we obtain the inequality

$$
\frac{\partial F(\mu, T)}{\partial \mu} \leq \frac{2 \beta e^{2 \beta\left(\mu_{c}-\mu\right)}}{\left(e^{2 \beta\left(\mu_{c}-\mu\right)}-1\right)^{2}}
$$

for $\mu<\mu_{c}$.

Till now we have already had some basic knowledge about the function $F(\mu, T)$, i.e., it should satisfy (7), (10), (13), (15) and (16) simultaneously. Under these conditions, we can solve equation (13) exactly, and the solution, being surprisingly simple, is given by

$$
F(\mu, T)=\frac{C(T)}{1+e^{2 \beta\left(\mu_{c}-\mu\right)}},
$$

where $C(T)$ is a positive, finite constant at temperature $T$, satisfying

$$
0<C(T) \leq 1
$$

We like to mention here that we can not obtain the closed form of $C(T)$ for the time being, but such a form is enough for our purpose. Consequently, by Eq.(7), we have

$$
\rho(\mu, T)=1+C(T) \tanh \left[\beta\left(\mu-\mu_{c}\right)\right]
$$

One may check that the solutions given by Eqs.(17) and (19) satisfy all aforementioned properties of functions $F(\mu, T)$ and $\rho(\mu, T)$.

With these facts, we are ready to address the problem of phase separation away from half-filling. As we have obtained an exact, explicit expression for $\rho(\mu, T)$, say, Eq.(19), it is obvious that $\rho(\mu, T)$ as a function of $\mu$ is continuous at any fillings. This result shows that there is no occurrence of the first-order phase transition, which in turn leads to the conclusion that the one-band Hubbard model can not exhibit the phenomenon of phase separation on a square lattice at finite temperature, consistent with the numerical calculations [8,4].

A few remarks concerning Eqs.(17) and (19) are in order. (1) As one may note that, Eq.(19) looks to be reasonable, as it comes directly from the symmetry of the Hubbard model, and is well in agreement with the inequality (6). Moreover, $\rho(\mu, T)$, given by (19), has the qualitatively similar behaviors compared with numerical results on square lattices with small sizes, though a quantitative comparison is impossible due to uncertainty of $C(T)$ as well as the lack of accurate data. Clearly, Eq.(19) remains valid in the thermodynamic limit. We like to mention here that we can not prove the uniqueness of the solution Eq.(17), but the other solutions, if exist, might have the similar forms as Eq.(17) [14], which would not affect our conclusion. (2) As indicated in Eq.(19), $\rho(\mu, T)$ might have different behaviors for positive and negative $U$ because of possible $\mu_{c}$-dependence of $C(T)$. (3) We emphasize once again that Eqs.(17) and (19) work only for finite temperatures and finite chemical potentials. One can not extract any useful information for the zero-temperature case from this study. Based upon the present result, however, we could say that it may be inappropriate to use the one-band Hubbard model as a model to explain the phenomena of phase separation observed in high Tc superconductors, at least at finite temperatures.

In summary, we show in a somewhat exact way that, using the known exact results and symmetry properties for the one-band Hubbard model, there is no phase separation at any fillings on a square lattice at finite temperature for both negative and positive $U$. This result is consistent with the quantum Monto Carlo calculation and some analytic result, and is also compatible with the recent exact result obtained in Ref. [5]. Nevertheless, we like to mention that our result does not cover the case at zero temperature. As there are also strong numerical evidences showing no occurrence of phase separation at zero temperature, how to obtain an exact proof for both $U>0$ and $U<0$ is still a fascinating topic.

\section{ACKNOWLEDGMENTS}

The author is indebted to Dr. A. Schadschneider and Prof. B.H. Zhao for various discussions. He is also grateful to Prof. J. Zittartz and ITP of Universität zu Köln for the warm hospitality, and to the Alexander von Humboldt Stiftung for financial support. 
* On leave of absence from Graduate School, Chinese Academy of Sciences, Beijing, China. E-mail: gs@thp.uni-koeln.de.

[1] J.D. Jorgensten, B. Dabrowski et al, Phys. Rev. B38, 11337(1988); D.R. Harshman, G. Aeppli et al, Phys. Rev. Lett. 63, 1187(1989).

[2] E.L. Nagaev, Phys. -Uspek. 38, 497(1995) and references therein.

[3] For example, see Phase Separation in Cuprate Superconductors, eds. E. Sigmund and K.A. Müller, (SpringerVerlag, Berlin, 1994).

[4] E. Dagotto, Rev. Mod. Phys. 66, 763(1994) and references therein.

[5] Chi Au, B.H. Zhao and H.T. Nieh, Phys. Rev. Lett., to be published. Report-No. cond-mat/9602054.

[6] E.H. Lieb, Phys. Rev. Lett. 62, 1201(1989).
[7] K. Kubo and K. Kishi, Phys. Rev. B41, 4866(1990).

[8] A. Moreo and D.J. Scalapino, Phys. Rev. Lett. 66, 946(1991); A. Moreo, D.J. Scalapino and E. Dagotto, Phys. Rev. B43, 11442(1991); E. Dagotto et al., Phys. Rev. B45, 10741(1992); N. Furukawa and M. Imada, J. Phys. Soc. Jpn. 61, 3331(1992); etc.

[9] D.K. Ghosh, Phys. Rev. Lett. 27, 1584(1971).

[10] W.O. Putikka, M.U. Luchini and T.M. Rice, Phys. Rev. Lett. 68, 538(1992).

[11] C.N. Yang, Phys. Rev. Lett. 63, 2144(1989); C.N. Yang and S.C. Zhang, Mod. Phys. Lett. B4, 759(1990).

[12] G. Su and B.H. Zhao, Phys. Lett. A200, 191(1995).

[13] H. Shiba, Prog. Theor. Phys. 48, 2171(1972).

[14] A. Schadschneider, private communication. 